\title{
Anti-synthetase syndrome: a rare and challenging diagnosis for bilateral ground-glass opacities-a case report with literature review
}

\author{
Nasam Alfraji ${ }^{*}$, Usman Mazahir ${ }^{2}$, Moiuz Chaudhri ${ }^{2}$ and Jeffrey Miskoff ${ }^{2}$
}

\begin{abstract}
Background: Anti-synthetase syndrome (ASS) is an uncommon immune-mediated entity characterized by myositis, interstitial lung disease (ILD), non-erosive arthritis, and less common features such as fever, Raynaud's phenomenon, and skin changes in association with anti-aminoacyl-transfer-RNA antibodies, most commonly anti-Jo-1 antibodies.

Case presentation: We present a challenging and rare case of ASS-associated ILD presenting with unexplained respiratory symptoms and bilateral infiltrates on chest imaging during the COVID-19 pandemic. High clinical suspicion for ASS with early appropriate therapy with corticosteroids and immunosuppressive agents led to marked clinical improvement.

Conclusion: High index of suspicion for ASS is mandated in patients with unexplained ILD. A comprehensive autoimmune work-up is important as an early treatment with corticosteroids with or without immunomodulators improves patient outcomes and survival in an otherwise poor prognostic disease.
\end{abstract}

Keywords: Anti-synthetase syndrome, Autoimmune disease, Interstitial lung disease, Corticosteroids

\section{Background}

Anti-synthetase syndrome (ASS) is a rare multisystemic autoimmune disease with variable manifestations ranging from myositis, interstitial lung disease (ILD), and non-erosive arthritis to less common features such as fever, Raynaud 's phenomenon, and skin changes in the setting of detectable anti-aminoacyl-transfer-RNA antibodies, most commonly anti-Jo-1 antibodies [1].

ASS has unclear pathogenesis; however, it is hypothesized that immune intolerance and immune self-reactivity have major implications [2, 3]. It is thought to develop in genetically susceptible individuals in the setting of

\footnotetext{
*Correspondence: nasam.alfraji@hmhn.org

${ }^{1}$ Department of Internal Medicine, Jersey Shore University Medical

Center, Hackensack Meridian Health, Neptune, NJ 07753, USA

Full list of author information is available at the end of the article
}

triggering environmental factors and immune system activation $[2,4]$.

ASS is considered by some authors as a separate disease entity within the idiopathic inflammatory myopathy (IIM) besides dermatomyositis (DM) and polymyositis (PM) [2]. ASS occurs mainly in adults with average age 50 years and more commonly in Caucasian females than males $[5,6]$.

The prevalence of ILD has been reported in $69-100 \%$ of ASS patients in several studies which is also the major predictor of mortality and morbidity. ILD is the presenting feature of ASS in only $15-30 \%$. Patients usually present with exertional dyspnea with or without dry cough. Pulmonary hypertension also reported with or without concomitant ILD [2, 6].

ASS is considered a challenging and under-recognized clinical entity that requires a high index of suspicion as it 
can mimic other diseases such as infections especially if presenting with incomplete disease pattern [7]. Early recognition, monitoring for new disease manifestations, and treatment with corticosteroids and other immunosuppressive agents result in a better outcome and prognosis [7].

We inhere report a rare case of ASS with a primary and an isolated ILD, which presented during the COVID-19 pandemic era rendering the diagnosis more challenging. This case has been reported in line with the CARE criteria.

\section{Case presentation}

A 51-year-old white female who was referred from our outpatient pulmonary clinic to the ED (emergency department) for worsening respiratory distress and six weeks history of persistent dry cough. Prior to this admission, the patient was seen at an urgent care and was prescribed a 10 days course of oral antibiotic empirically without significant improvement. Subsequently, the patient was seen in our outpatient pulmonary clinic for a working diagnosis of pneumonia and a previous chest $x$-ray depicting bibasilar opacities. In the office, the patient complained of dyspnea with ambulation. She was tested negative for COVID-19. Therefore, patient was prescribed oral doxycycline $100 \mathrm{mg}$ twice daily empirically for 14 days and $40 \mathrm{mg}$ of oral prednisone for 5 days. The patient was advised to communicate her progress with the treating physician, so her treatment protocol can be adjusted if needed. However, attempts to wean her prednisone made her dyspnea worse with pulse oximetry reportedly in high $80 \mathrm{~s} \%$ on ambulation. PFTs (pulmonary function tests) outpatient showed restrictive lung pattern with reduced FEV-1 (forced expiratory volume in $1 \mathrm{~s}$ ) at $52 \%$, and reduced FVC (Forced vital capacity) at 47\%.

Patient reported having associated generalized fatigue, but no weight loss. She denied any hemoptysis, chest pain, fever, chills, night sweats, epistaxis, dry eyes, dry mouth, vision changes, photosensitivity, oral ulcer, dysphagia, abdominal pain, nausea, vomiting, constipation, or diarrhea. She denied any urinary disturbances, myalgia, joint pain or swelling, blood in urine or stool, or any Raynaud's type symptoms. Patient reported a recent travel history to Florida, but no history of sick contacts. She endorsed a family history only significant for Crohn's disease in father and daughter. She reported no alcohol use and no smoking history. The patient did not have any occupational or pet exposure.

In the ED, the patients' vital signs were a temperature of $97.6 \mathrm{~F}$, respiratory rate of 26 per minute, oxygen saturation of $88-90 \%$ on room air which improved to $94 \%$ with $3 \mathrm{~L}$ of oxygen on nasal cannula, and blood pressure of 102/53 mmHg. Patient's body weight was $75.3 \mathrm{~kg}$ and her height was $157 \mathrm{~cm}$. On physical exam, patient noticed to be dyspneic and tachypneic. She was alert and oriented to time, place and person. Head and neck examinations were unremarkable for lymphadenopathy, jugular venous distention (JVD), nasal/oral ulcerations, or any other lesions. On chest auscultation, reduced breath sounds were evident at the lower lung fields. Cardiology, gastrointestinal, and neurological examinations were unremarkable. No joint tenderness/swelling or muscle tenderness/weakness appreciated at the musculoskeletal examination. Skin examination revealed no rash or other lesions. The patient was admitted for further evaluation, beginning with retesting for COVID-19 and additional workup.

Initial complete blood count, renal, and liver panel were all within normal limits except for normocytic anemia (Table 1). Erythrocyte sedimentation rate (ESR) was $49 \mathrm{~mm} / \mathrm{h}(0-15 \mathrm{~mm} / \mathrm{h}), \mathrm{C}$-reactive protein (CRP) was $3.24 \mathrm{mg} / \mathrm{dL}(0.00-0.74 \mathrm{mg} / \mathrm{dL})$, and anti-nuclear antibodies 2.09 (0.0-0.90). Urinalysis and complement levels were unremarkable. Creatine kinase $(\mathrm{CK})$ and aldolase were $326 \mathrm{iu} / \mathrm{L}(22-232 \mathrm{iu} / \mathrm{L}), 39.5 \mathrm{u} / \mathrm{L}(1.5-8.1 \mathrm{u} / \mathrm{L})$ respectively. The COVID-19 RT-PCR (Reverse transcription polymerase chain reaction) testing was negative twice as an inpatient.

A chest x-ray (Fig. 1) showed persistent bibasilar infiltrates, finding similar to previous imaging. A Computed tomography angiography of the chest (Fig. 2) showed bilateral ground glass opacities, with shotty mediastinal lymph nodes, and no filling defects to suggest a pulmonary embolism. Echocardiogram showed normal left ventricular (LV) function, right ventricular function, and pulmonary pressures.

The patient underwent bronchoscopy with right lower lobe transbronchial biopsies which showed mild lung parenchymal inflammation, fibrosis, and reactive epithelial changes without any sign of malignancy. Gomori methenamine-silver (GMS) nitrate stain and acid-fast stains (also known as the Ziehl-Neelsen stain) were negative for Pneumocystis jiroveci and Mycobacterium species, respectively. Bacterial and fungal cultures from right lower lobe bronchoalveolar lavage remained negative.

Subsequent autoimmune screening returned strongly positive for anti-Jo-1 antibody $191 \mathrm{au} / \mathrm{mL}$ (reference range $0-40$ ). Other markers, including rheumatoid factor, anti-cyclic citrullinated peptide antibodies, anti-Ro/ SSA, and antineutrophil cytoplasmic antibodies were negative.

Therefore, ASS-associated ILD was considered in the setting of clinical and radiographic findings of nonspecific interstitial pneumonia (NSIP) associated with positive anti-Jo-1 antibody. 
Table 1 Summary of main laboratory investigations at admission and follow-up

\begin{tabular}{|c|c|c|c|}
\hline Lab & Admission & 3-months follow-up & Reference value \\
\hline Hemoglobin & 9.8 & 12.7 & $12-16 \mathrm{~g} / \mathrm{dL}$ \\
\hline WBC & 10.3 & 6.2 & $4.5-11.0 \mathrm{~K} / \mathrm{uL}$ \\
\hline Platelets & 285 & 325 & $140-450 \mathrm{~K} / \mathrm{uL}$ \\
\hline BUN & 4 & - & $5-25 \mathrm{mg} / \mathrm{dL}$ \\
\hline Creatinine & 0.32 & - & $0.61-1.24 \mathrm{mg} / \mathrm{dL}$ \\
\hline Glomerular filtration rate & $>60$ & - & $>60$ \\
\hline ALT & 46 & 14 & $10-60 \mathrm{U} / \mathrm{L}$ \\
\hline AST & 57 & 16 & $10-42 \mathrm{U} / \mathrm{L}$ \\
\hline ESR & 49 & 22 & $0-15 \mathrm{~mm} / \mathrm{h}$ \\
\hline CRP & 3.24 & 0.45 & $0.00-0.74 \mathrm{mg} / \mathrm{dL}$ \\
\hline Rheumatoid factor & $<20$ & - & $<20 \mathrm{U} / \mathrm{mL}$ \\
\hline Anti-nuclear antibodies & 2.09 & - & $0.0-0.90$ \\
\hline C3 & 106 & - & $85-170 \mathrm{mg} / \mathrm{dL}$ \\
\hline C4 & 22.2 & - & $16-40$ mg/dL \\
\hline JO-1 antibody & 191 & - & $0-40 \mathrm{AU} / \mathrm{mL}$ \\
\hline c-ANCA & $<1.20$ & - & $<1.20$ \\
\hline p-ANCA & 0.0 & - & $0-19 \mathrm{U} / \mathrm{mL}$ \\
\hline Anti-dsDNA & $<1: 10$ & - & $<1: 10$ \\
\hline Scleroderma (scl-70) antibody & 3.0 & - & 0-40 AU/mL \\
\hline Anti-Smith antibody & 1.0 & - & $0-40 \mathrm{AU} / \mathrm{mL}$ \\
\hline Anti-Sjögren's-syndrome-related Ag A & 5.0 & - & 0-40 AU/mL \\
\hline Anti-Sjögren's-syndrome-related Ag B & 1.0 & - & $0-40 \mathrm{AU} / \mathrm{mL}$ \\
\hline Anti-RNP antibody & 8.0 & - & 0-40 AU/mL \\
\hline Anti-cyclic citrullinated antibodies & 3.0 & - & $0-19$ units \\
\hline Legionella antigen, urine & Negative & - & Negative \\
\hline Streptococcus pneumonia antigen & Negative & - & Negative \\
\hline Beta-D-glucan & Negative & - & Negative \\
\hline Procalcitonin & 0.11 & - & $<0.50 \mathrm{ng} / \mathrm{mL}$ \\
\hline Aldolase & 39.5 & 4.5 & $1.5-8.1 \mathrm{u} / \mathrm{L}$ \\
\hline Creatine kinase & 326 & 58 & $22-232 \mathrm{iu} / \mathrm{L}$ \\
\hline
\end{tabular}

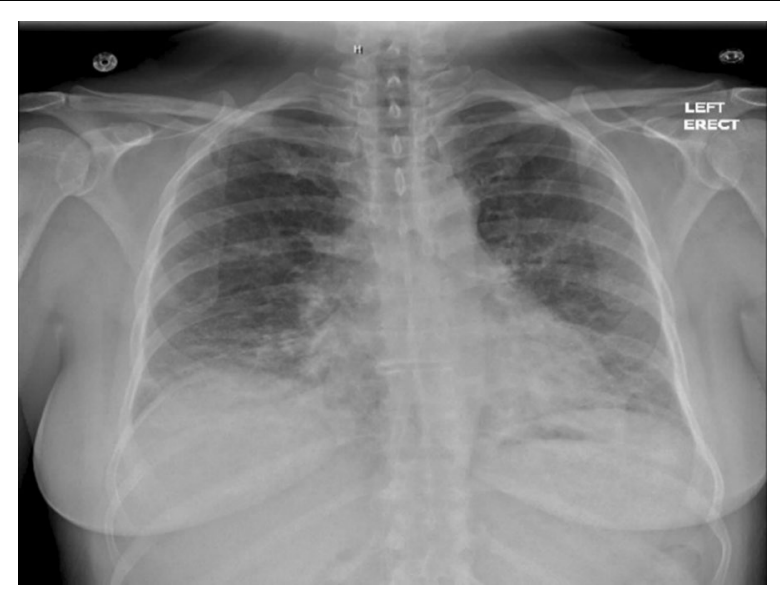

Fig. 1 Chest $x$ ray revealing persistent bibasilar infiltrates

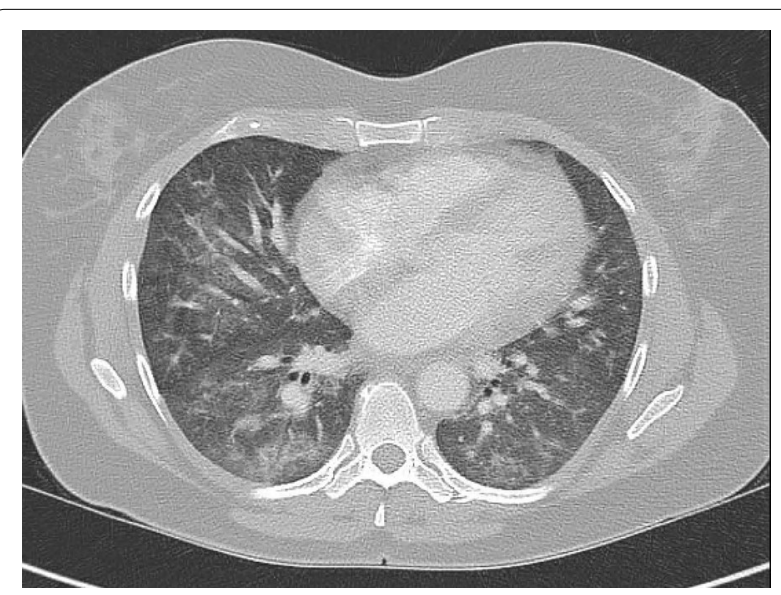

Fig. 2 Computed tomography angiography of the chest revealing bilateral ground glass opacities, with no filling defects suggesting pulmonary emboli 
Patient was started on intravenous methylprednisolone $40 \mathrm{mg}$ every $12 \mathrm{~h}$ which failed to improve patient's symptoms; therefore, she was given intravenous pulse methylprednisolone $1000 \mathrm{mg}$ daily for 3 days. Patient reported some improvement of her symptoms after pulse steroids. She was discharged with $2 \mathrm{~L}$ home oxygen as needed and on high dose oral corticosteroids, prednisone $60 \mathrm{mg}$ oral daily. She followed up with rheumatology outpatient two weeks after discharge and was started on oral mycophenolate $500 \mathrm{mg}$ twice daily. However, tapering steroids was difficult and mycophenolate was titrated up to $1500 \mathrm{mg}$ twice daily.

At her 3 months follow-up, the patient continued to have a gradual improvement of her symptoms and she was weaned off oxygen. Chest high-resolution computed tomography (HRCT) at that time showed 20\% interval improvement particularly in the lower lobes with improvement of her laboratory markers such as ESR, CRP, CK, and aldolase demonstrated in (Table 1, column 2). Favorably, her prednisone was tapered over six months to $10 \mathrm{mg}$ daily while being on the same dose of mycophenolate.

\section{Discussion}

Anti-synthetase syndrome (ASS) is a rare autoimmune disease characterized mainly by interstitial lung disease (ILD), myositis, and arthritis reportedly in $90 \%$ of cases $[2,8]$. However, other manifestations like fever, rashes, and Raynaud's syndrome have also been seen less commonly [2, 8]. ILD was noted to be the initial presentation in $15-30 \%$ of ASS patients in various studies [2]. The main serological hallmark of this syndrome is the presence of various autoantibodies to aminoacyl transfer RNA (tRNA) synthetase including Jo-1 and others [2]. ASS was first identified as a clinical entity by Marguerie et al. in 1990 [6].

The complete form of ASS includes the triad of ILD, myositis, and arthritis which is only reported in 19.5\% at disease onset in Cavagna et al. study and lacking any of these features is considered as an incomplete form of ASS [9]. Patient with incomplete picture might eventually exhibit other manifestations to have a complete form over a variable period of time [9].

In 2016, Trallero-Araguás et al. studied the clinical manifestations and long-term outcome of 148 patients with anti-Jo-1 syndrome from 18 Spanish hospitals. Most of the cases had an incomplete picture of Anti-synthetase syndrome at onset as follows: isolated ILD 47 (32.4\%) patients, isolated myositis 39 (26.9\%) patients, and isolated polyarthritis $26(17.9 \%)$ patients. Only a minority had stable disease at the end of follow-up with isolated ILD still reported in 21 (14.5\%) of patients, isolated myositis in 23 (15.9\%) and isolated polyarthritis in three
(2.1\%) patients [10]. Mortality rate was reportedly fourfold higher than general population [10].

There are still no unified internationally-recognized ASS classification criteria; however, there are two main classification criteria for ASS, Connors et al. 2010, and Solomon et al. 2011, and both require serological and specific clinical features [2]. Our case was diagnosed in accordance with Connors et al. 2010 criteria that require the presence of anti-synthetase antibodies with one or more of the following: myositis by Bohan and Peter criteria, ILD not explained by other causes, arthritis, persistent fever, Raynaud's phenomenon, and mechanic's hands [2].

ILD is the main pulmonary manifestation of ASS and the major cause of morbidity and mortality [2]. Patients usually present with dyspnea on exertion accompanied by dry cough [6]. Instrumentally, ILD is defined by PFTs (pulmonary function tests) which show restrictive picture, indicated by total lung capacity (TLC) $<80 \%$ predicted, diffusion capacity of lung for carbon monoxide (DLCO) $<70 \%$ of predicted value, and/or forced vital capacity $(\mathrm{FVC})<80 \%$ predicted, and/ or specific findings on HRCT scans of the lungs $[2,5]$. The usual type of ILD seen on HRCT is nonspecific interstitial pneumonia (NSIP) which characterized by ground-glass opacities. Other types include COP (cryptogenic organizing pneumonia) characterized by consolidation and linear opacities, and usual interstitial pneumonia (UIP) characterized by honeycomb pattern and traction bronchiectasis [5, 11]. Bronchoscopy and lung biopsy are not indicated routinely for diagnosis of ASS; however, it could be considered to rule out other causes or exclude infection [2].

There is still no standardized regimen for ASS treatment; however, treatment with prednisone $1 \mathrm{mg} / \mathrm{kg} /$ day (or methylprednisolone $1 \mathrm{~g}$ IV daily for 3 days, in severe cases) is the most common initial therapy [2]. Additionally, a steroid-sparing agent (usually mycophenolate or azathioprine) can be used along with steroid and has showed a better survival and fewer relapses in comparison to prednisone monotherapy [2]. Other case studies reported using cyclophosphamide, cyclosporine, and methotrexate, and intravenous immunoglobulins as immunomodulating agents $[1,5,7]$. Rituximab has been used for patients with refractory ILD [8].

Several prognostic factors of poor outcome and reduced survival have been identified in ASS including old age, African-American ethnicity, Male gender, UIP picture on HRCT chest, symptomatic ILD, steroid-resistant ILD, lower numbers of FVC and DLCO at time of diagnosis $[1,7,8]$. However, the severity of ILD remains the ultimate prognostic factor of ASS $[1,5]$.

The association between ASS and malignancy is controversial. Although some studies including one large 
cohort study reported no increase in malignancy rates in ASS patients comparing to the general population, other case series and reports mentioned development of different malignancies within variable period of ASS diagnosis $[2,5,12]$. In one of the case series, malignancy involved the breast, stomach, cervix, thyroid, nasopharynx, and salivary duct developed in 8 (6.5\%) of 124 patients [12]. Therefore, authors recommend age-appropriate malignancy screening for all patients with ASS $[2,5,12]$.

Our unique case was a rare clinical entity which posed a diagnostic challenge as patient presented with worsening dyspnea and dry cough at the onset of COVID-19 pandemic. However, carrying a high index of suspicion and performing a comprehensive work-up led to an early diagnosis of ASS and prompt treatment resulting in a favorable outcome. Our patient showed a fair improvement after intravenous pulse steroids dosing and was started on high dose oral prednisone. Our decision to add a second immunosuppressive agent was based on our patient's progressive presentation with respiratory symptoms and exertional hypoxia along with the presence of anti-synthetase antibodies. Furthermore, a steroid-sparing agent (mycophenolate in our case) has been chosen as it showed fewer relapses in comparison to prednisone monotherapy in several studies [2].

\section{Conclusion}

ASS is a rare clinical syndrome with a variable symptomology and combination. Our case emphasizes the importance of considering ASS-associated ILD in our differentials in case of unexplained respiratory symptoms with bilateral grand-glass opacities on chest imaging after excluding other possibilities including infections as early diagnosis and timely treatment would improve patient outcome and survival. Although our case had clinical improvement with corticosteroids treatment and subsequently immunosuppressive agent, there are still no enough standardized guidelines about the management of ASS-associated ILD. Therefore, it requires more randomized controlled trials and research with larger sample size to mainstream the approach for diagnosing and treating ASS.

\footnotetext{
Abbreviations

ASS: Anti-synthetase syndrome; ILD: Interstitial lung disease; IIM: Idiopathic inflammatory myopathy; DM: Dermatomyositis; PM: Polymyositis; PFTs: Pulmonary function tests; JVD: Jugular venous distention; ESR: Erythrocyte sedimentation rate; CRP: C-reactive protein; CK: Creatine kinase; RT-PCR: Reverse transcription polymerase chain reaction; TLC: Total lung capacity; LV: Left ventricular; GMS: Gomori methenamine-silver; NSIP: Nonspecific interstitial pneumonia; DLCO: Diffusion capacity of lung for carbon monoxide; COP: Cryptogenic organizing pneumonia; UIP: Usual interstitial pneumonia; HRCT: High-resolution computed tomography.
}

Acknowledgements

No acknowledgments are made.

\section{Authors' contributions}

$J M$ and UM: were involved in the patient care and case selection. NA: was involved in writing and reviewing the abstract, the introduction, the discussion, and the conclusion of the manuscript. NA and MC: was involved in writing and reviewing the case description part of the manuscript. UM and JM: worked on the planning, manuscript revision and final approval. All authors read and approved the final manuscript.

\section{Funding}

The authors did not obtain funding for the manuscript.

\section{Availability of data and materials}

Data sharing is not applicable to this article as no datasets were generated or analyzed during the current study.

\section{Ethics approval and consent to participate}

The authors have no statements to disclose for this publication.

\section{Consent for publication}

The patient described in the case report had given informed written consent for the publication of the case report including any identifying images, and any personal or clinical details.

\section{Competing interests}

The authors declare that they have no competing interests.

\section{Author details}

${ }^{1}$ Department of Internal Medicine, Jersey Shore University Medical Center, Hackensack Meridian Health, Neptune, NJ 07753, USA. ${ }^{2}$ Department of Pulmonology and Critical Care, Jersey Shore University Medical Center, Neptune, NJ 07753, USA.

Received: 18 December 2020 Accepted: 21 December 2020

Published online: 06 January 2021

\section{References}

1. Devi HG, Pasha MM, Padmaja MS, Halappa S. Anti-synthetase syndrome: a rare cause for ILD. J Clin Diagn Res. 2016;1 0(3):OD08-9. https://doi. org/10.7860/JCDR/2016/16872.7361.

2. Marco $\mathrm{JL}$, Collins BF. Clinical manifestations and treatment of antisynthetase syndrome. Best Pract Res Clin Rheumatol. 2020;10:101503. https ://doi.org/10.1016/j.berh.2020.101503.

3. Gallay L, Gayed C, Hervier B. Antisynthetase syndrome pathogenesis: knowledge and uncertainties. Curr Opin Rheumatol. 2018;30(6):664-73. https://doi.org/10.1097/BOR.0000000000000555.

4. O'Hanlon TP, Carrick DM, Targoff IN, Arnett FC, Reveille JD, Carrington $M$, et al. Immunogenetic risk and protective factors for the idiopathic inflammatory myopathies: distinct HLA-A, -B, -CW, -DRB1, and -DQA1 allelic profiles distinguish European American patients with different myositis autoantibodies. Medicine (Baltim). 2006;85(2):111-27. https:// doi.org/10.1097/01.md.0000217525.82287.eb.

5. Badshah A, Haider I, Pervez S, et al. Anti-synthetase syndrome presenting as interstitial lung disease: a case report. J Med Case Rep. 2019;13:241. https://doi.org/10.1186/s13256-019-2146-0.

6. Khan KA, Ahmed WN. Anti-synthetase syndrome: A case report and literature review. Hamdan Med J. 2018;11:87-90. https://doi.org/10.4103/ HMJ.HMJ_17_18.

7. Rüegg CA, Maurer B, Laube I, et al. Jo1-antisynthetase syndrome and severe interstitial lung disease with organising pneumonia on histopathology with favourable outcome on early combined treatment with corticosteroids, mycophenolate mofetil and rituximab. BMJ Case Rep. 2019;12:e231006. https://doi.org/10.1136/bcr-2019-231006.

8. Monti S, Montecucco C, Cavagna L. Clinical spectrum of anti-Jo-1-associated disease. Curr Opin Rheumatol. 2017;29(6):612-7. https://doi. org/10.1097/BOR.0000000000000434. 
9. Cavagna L, Nuno L, Scire CA, Govoni M, Longo FJL, Franceschini F, et al. Clinical spectrum time Course in anti Jo-1 positive anti-synthetase syndrome. Medicine (Baltim). 2015;94(32):e1144. https://doi.org/10.1097/ MD.0000000000001144.

10. Trallero-Araguas E, Grau-Junyent JM, Labirua-Iturburu A, et al. Clinical manifestations and long-term outcome of anti-Jo1 antisynthetase patients in a large cohort of Spanish patients from the GEAS-IIM group. Semin Arthritis Rheum. 2016;46:225-31. https://doi.org/10.1016/j.semar thrit.2016.03.011.

11. Razek AAKA, El Badrawy MK, Alnaghy E. Interstitial lung fibrosis imaging reporting and data system: what radiologist wants to know? J Comput
Assist Tomogr. 2020;44:656-66. https://doi.org/10.1097/RCT.0000000000 001075.

12. Shi J, Li S, Yang H, et al. Clinical profiles and prognosis of patients with distinct anti-synthetase autoantibodies. J Rheumatol. 2017:44:1051-7. https://doi.org/10.3899/jrheum.161480.

\section{Publisher's Note}

Springer Nature remains neutral with regard to jurisdictional claims in published maps and institutional affiliations.
Ready to submit your research? Choose BMC and benefit from:

- fast, convenient online submission

- thorough peer review by experienced researchers in your field

- rapid publication on acceptance

- support for research data, including large and complex data types

- gold Open Access which fosters wider collaboration and increased citations

- maximum visibility for your research: over $100 \mathrm{M}$ website views per year

At BMC, research is always in progress.

Learn more biomedcentral.com/submissions 